\title{
BMJ Global Health Institutional mistrust and child vaccination coverage in Africa
}

\author{
Nik Stoop (1) , ${ }^{1,2,3}$ Kalle Hirvonen (D) , ${ }^{4}$ Jean-Francois Maystadt (iD) 5,6,7
}

To cite: Stoop N, Hirvonen K, Maystadt J-F. Institutional mistrust and child vaccination coverage in Africa. BMJ Global Health 2021;6:e004595. doi:10.1136/ bmjgh-2020-004595

Handling editor Senjuti Saha

- Additional supplemental material is published online only. To view, please visit the journal online (http://dx.doi.org/10. 1136/bmjgh-2020-004595)

Received 3 December 2020 Revised 14 March 2021 Accepted 11 April 2021

\section{Check for updates}

(c) Author(s) (or their employer(s)) 2021. Re-use permitted under CC BY-NC. No commercial re-use. See rights and permissions. Published by BMJ.

For numbered affiliations see end of article.

\section{Correspondence to} Dr Jean-Francois Maystadt; jean-francois.maystadt@ uclouvain.be

\section{ABSTRACT}

Introduction Despite considerable improvements in vaccination coverage over the last decade, half of the world's unvaccinated and undervaccinated children are located in Africa. The role of institutional trust in explaining vaccination gaps has been highlighted in several qualitative reports but so far has only been quantified in a small number of high-income countries.

Methods We matched information on child vaccination status from the Demographic Health Surveys with information on institutional trust from the Afrobarometer surveys at the subnational level. A total of 166953 children from 41 surveys administered in 22 African countries covering 216 subnational regions were used. Based on a principal component analysis, we constructed an institutional mistrust index that combined the level of mistrust in the head of state, parliament, electoral system, courts and local government. Associations between institutional mistrust and child vaccination uptake were assessed with multivariable fixed effects logistic regressions that controlled for time-invariant subnational region characteristics and various child, caregiver, household and community characteristics.

Results A 1 SD increase in the institutional mistrust index was associated with a $10 \%(95 \% \mathrm{Cl}$ of ORs: 1.03 to 1.18$)$ increase in the likelihood that a child had not received any of eight basic vaccines and with a $6 \%$ decrease in the likelihood a child had received all of the basic vaccines (95\% Cl: 0.92 to 0.97$)$. Institutional mistrust was negatively associated with the likelihood that a child had received each of the eight basic vaccinations $(p<0.05)$

Conclusions Child vaccination rates in Africa are considerably lower in areas in which the local population displays high levels of mistrust towards local authorities. Institutional mistrust is an important dimension of vaccine hesitancy, considered as one of the most important threats to global health. Empowering local authorities with resources and communication strategies to address institutional mistrust may be needed to close the remaining vaccination gaps in Africa.

\section{INTRODUCTION}

Immunisation is one of the most cost-effective public health interventions to prevent child mortality and morbidity. ${ }^{1}$ The adoption of the Global Vaccine Action Plan in 2012 provided a roadmap to promote equitable access to vaccines and to reach the Sustainable Development Goals (SDG) towards Universal

\section{Key questions}

What is already known?

- Evidence from a small number of high-income countries shows how parents who report low trust in national authorities are less likely to vaccinate their children.

- Qualitative studies conducted in Africa similarly suggest that mistrust towards local and national authorities could be an important contributor to vaccine hesitancy.

- Overall, the available evidence on the role of trust in public institutions influencing vaccination uptake is scarce with limited attention to confounding factors that may affect both vaccination uptake and public trust towards local authorities.

\section{What are the new findings?}

- To our knowledge, this is the first comprehensive attempt to quantify the role of institutional mistrust on child vaccination uptake in low-income and middleincome countries.

- Using multivariable subnational region fixed effects logistic regressions, we found that a 1 SD increase in institutional mistrust in a given subnational region was associated with a $10 \%$ increase in the likelihood that a child had not received any of eight basic vaccines and with a $6 \%$ decrease in the likelihood a child had received all of the basic vaccines.

\section{What do the new findings imply?}

- Our findings indicate that institutional mistrust is an important barrier in reaching universal child immunisation in Africa - the region that is most lagging behind in achieving universal child vaccination coverage.

- Low levels of institutional trust pose a significant risk to vaccination campaigns and call for empowering local authorities with resources and effective communication strategies.

Health Coverage. ${ }^{2}$ The last two decades have shown progress in vaccination coverage that has translated into a global decline in the incidence of and mortality from infectious diseases. ${ }^{3}$ In the last decade, however, progress has stalled. The share of children receiving three doses of diphtheria-tetanuspertussis-containing vaccines, for instance, 
has plateaued at $85 \%$ while the incidence of measles has increased. ${ }^{3}$

Despite remarkable progress over the last two decades in improving vaccination coverage, Africa continues to lag behind. At the end of 2018, nearly half of the world's unvaccinated and undervaccinated children were located in Africa. ${ }^{4}$ Moreover, two-thirds of the 53 countries that are not in the course of meeting the SDG target of reducing under-5 mortality to at least 25 per 1000 live births by 2030 are located in Africa. ${ }^{3}$ Within Africa, Eastern and Southern Africa have higher child vaccination coverages than West and Central Africa. ${ }^{5}$ However, more refined data reveal substantial inequalities both across and within countries. ${ }^{6-9}$ While the persistence of subnational pockets of low vaccination coverage in Africa has been attributed to societal, cultural and religious considerations as well as to limited access to vaccination services and to caregiver knowledge gaps, ${ }^{6} 1011$ a number of experts highlight the role of vaccine hesitancy in hindering progress towards universal child immunisation coverage. ${ }^{12-15}$ The Strategic Advisory Group of Experts on Immunisation defined vaccine hesitancy as 'a delay in acceptance or refusal of vaccination despite availability of vaccination services'. ${ }^{16}$

A key dimension of vaccine hesitancy relates to trust $^{17}{ }^{18}$; lack of trust in the vaccines, mistrust in the health service sector that provides them and mistrust in the authorities that decide on vaccination schedules and oversee the provision of health services. ${ }^{16}$ A systematic review of qualitative studies in low-income and middleincome countries reported that a lack of trust in vaccination programmes was the second most frequent concern leading to undervaccination. ${ }^{19}$ Similarly, a recent largescale quantitative analysis across 149 countries showed that vaccine uptake increases with confidence in the effectiveness and safety of vaccines, as well as confidence in medical advice provided by healthcare workers. ${ }^{15}$

Institutional mistrust-that is, the lack of trust in local and national authorities - has been documented to be an important contributor to vaccine hesitancy and refusal across high-income countries. ${ }^{20}{ }^{21}$ Lack of trust in governments has been found to lead parents to question the vaccine information they receive from health authorities and thus affect parents' decisions to vaccinate their children. ${ }^{19} 22$ The underlying intentions of the institutions involved in vaccination programmes are also being questioned..$^{20}$ For example, in some contexts, vaccine-hesitant parents widely believe that vaccine programmes are initiated to maximise profits of pharmaceutical companies that develop and distribute the vaccines. ${ }^{23}{ }^{24}$ Institutional mistrust is also related to concerns about vaccine safety, particularly among historically neglected and marginalised groups. ${ }^{2526}$

The role of institutional mistrust in explaining vaccine uptake in Africa has received less research attention. ${ }^{19} 27$ Qualitative evidence suggests that institutional mistrust is linked to vaccine hesitancy and refusal across the continent, including in Chad, ${ }^{28}$ the Democratic Republic of Congo, ${ }^{29}$ Mozambique, ${ }^{30}$ Nigeria ${ }^{31}$ and South Sudan. ${ }^{32}$
Apart from an insightful case study from the Democratic Republic of Congo during the Ebola disease epidemic, ${ }^{33}$ quantitative evidence on the role of institutional trust in vaccine hesitancy and uptake is rare. Therefore, in this study, we quantified the role of institutional mistrust on child vaccination uptake in 22 African countries.

\section{METHODS \\ Study design}

We matched child vaccination status with the degree of institutional trust measured at the first subnational administrative level in 22 African countries and assessed how changes in institutional trust were associated with changes in child vaccination status.

\section{Data sources}

The information on institutional trust was obtained from the Afrobarometer surveys periodically administered in 37 African countries since $1999 .^{34}$ The Afrobarometer surveys are representative at the national and the first subnational administrative levels. In Africa, the name of the first subnational administrative level varies between countries; for convenience, we therefore denote it by 'subnational region'. The surveys asked respondents how much they trust their relatives, people in general and public institutions of their country: the head of state, parliament, the electoral system, courts and local government.

Information on child vaccination status came from the Demographic and Health Surveys (DHS).$^{35}$ The DHS are nationally representative periodic surveys administered in most African countries. The surveys collected rich information on child health, including the vaccination status, for all children under 5 years of age. From DHS phase VII onwards, vaccination information was only collected for children under 3 years of age. Online supplemental appendix 1 provides more information and presents the child vaccination status for each survey included in the sample. The surveys further provided us with a wide range of variables at the child, parental, household and community level that could be correlated with child's vaccination status and institutional mistrust.

There were 22 countries with at least one overlapping Afrobarometer and DHS survey. We matched the two surveys using information on the subnational region, as described in online supplemental appendix 1. In total, we have data on 166953 children from 41 DHS surveys administered in 22 countries covering 216 subnational regions. The earliest DHS survey included in the study was administered in 2004 and the latest in 2018.

\section{Definitions of institutional trust and child vaccination status}

The institutional trust variables were measured at the subnational region level. Applying the Afrobarometer sampling weights, we calculated the share of population in each region that reported no trust in their head of state, parliament, electoral system, courts or local government. To account for the high correlation across these 
five dimensions of institutional trust, we applied a principal component analysis method to construct a single index of mistrust in public institutions (online supplemental appendix 2).

Following WHO guidelines, ${ }^{36}$ we excluded children younger than 12 months from the sample and focused on the eight basic child vaccinations: BCG; three doses of diphtheria, pertussis and tetanus; three doses of polio vaccine and one dose of measles-containing vaccine. The vaccination information in the DHS is first and foremost based on the information recorded on the vaccination card but if no card existed, caregiver's report on the child's vaccinations was used.

\section{Statistical analyses}

Our statistical approach exploited longitudinal variation in institutional trust within subnational regions. Our fixed effects regression model answered the question: 'What is the difference in child vaccination completion in a given subnational region between birth years when the level of institutional trust is low compared with another year when institutional trust is high?' We considered two binary outcome variables: one capturing children who had not received any of the eight basic vaccinations and one capturing children who had received all eight basic vaccinations. In additional analyses, we considered uptake of each of the eight basic vaccines as separate outcome variables. We modelled child's vaccination status as a function of the level of institutional mistrust in the subnational region at the time of the child's birth:

$$
\operatorname{prob}\left(Y_{i r t}=1\right)=\Phi\left(\beta D_{r t}+X_{i r t}^{\prime} \gamma+\theta_{r}+\tau_{t}\right)
$$

where $Y_{i r t}$ is the child's vaccination status, indexed for child $i$, subnational region $r$ and year of DHS survey t. The variable $\mathrm{D}$ is the exposure variable, an index measuring institutional mistrust in subnational region $r$ in which the child is located at the time of his/her birth $t$. Coefficient $\beta$ is estimated from the model and represents the conditional association between institutional mistrust and child's vaccination status. In additional analyses, we considered the different dimensions of institutional mistrust as exposure variables.

In line with similar multilevel studies, ${ }^{37-39}$ we kept the unit of analysis at the individual level to control for individual and household level characteristics. Vector $X$ includes several control variables at the child, parent, household and community level that could be correlated with child's vaccinations status and institutional mistrust. These variables include child's age (binary variables capturing age cohorts), sex and a birth-order and birthinterval variable; maternal age and age at which her first child was born; maternal and paternal level of education; household wealth, demographics and location (rural/ urban). We further controlled for access to and utilisation of healthcare services at the household and DHS cluster level. Online supplemental appendix 3 provides more information about these variables.
The subnational region fixed effects $\left(\theta_{r}\right)$ controlled for time-invariant unobserved heterogeneity within regions. As a result, $\beta$ was estimated from temporal variations within the subnational region in which the child resided at the time of his/her birth. The survey year fixed effects $\left(\tau_{t}\right)$ controlled for unobserved variation in the sample across DHS survey years. All models were estimated using logistic regressions. The subnational region and survey year fixed effects were accommodated using a dummy variable estimator approach. ${ }^{40}$ The coefficients were reported as ORs and CIs were calculated from SEs clustered at the subnational region level. We used Stata (V.15.1) for all statistical analyses.

We ran multiple robustness checks to assess the sensitivity of our findings. We considered alternative model specifications by varying the set of control variables and fixed effects. Information about maternal religion was not consistently available in DHS surveys and therefore not controlled for in the main regression models. To explore whether the association between institutional mistrust and child vaccination status is driven by maternal religion, we re-ran our regressions by adding maternal religion and restricting the sample to surveys in which this information was available. Moreover, both child vaccination status and institutional mistrust could be correlated with non-institutional forms of mistrust. To this end, we explored the relationship between child vaccination status and non-institutional mistrust indicators, including mistrust in people and a proxy for mistrust in medicine. For the latter, we relied on mother's refusal to consent to a free and non-invasive blood test for anaemia or HIV. Available in the DHS surveys, this variable has been considered as a revealed-preference measure of mistrust in medicine. ${ }^{41}$ Some children in the DHS were not born in the same cluster in which their household resided during the survey. To explore whether our findings were biased because of migrant households, we restricted the sample to children whose mother had lived in the same DHS cluster at least since the child was born. To explore the possibility that our findings were driven by a particular country (eg, due to its size or because of unusually high or low levels of institutional mistrust or child vaccination coverage) or a particular region in Africa, we re-ran our regressions by omitting each country and region at a time from the sample. Information on vaccination status was missing for $1.18 \%$ of eligible children; they were excluded from the sample. Following WHO guidelines,${ }^{36}$ we tested the robustness of our findings to including these children in the sample and treating them as not having received any vaccine. We further tested the robustness of our findings to the use of various sampling weights. Finally, we explored whether our findings also hold when aggregating the analysis at the level of the first subnational administrative level.

\section{RESULTS}

Summary statistics on child vaccination status and mistrust in public institutions are presented in table 1. 
Table 1 Summary statistics on child vaccination status and mistrust in public institutions

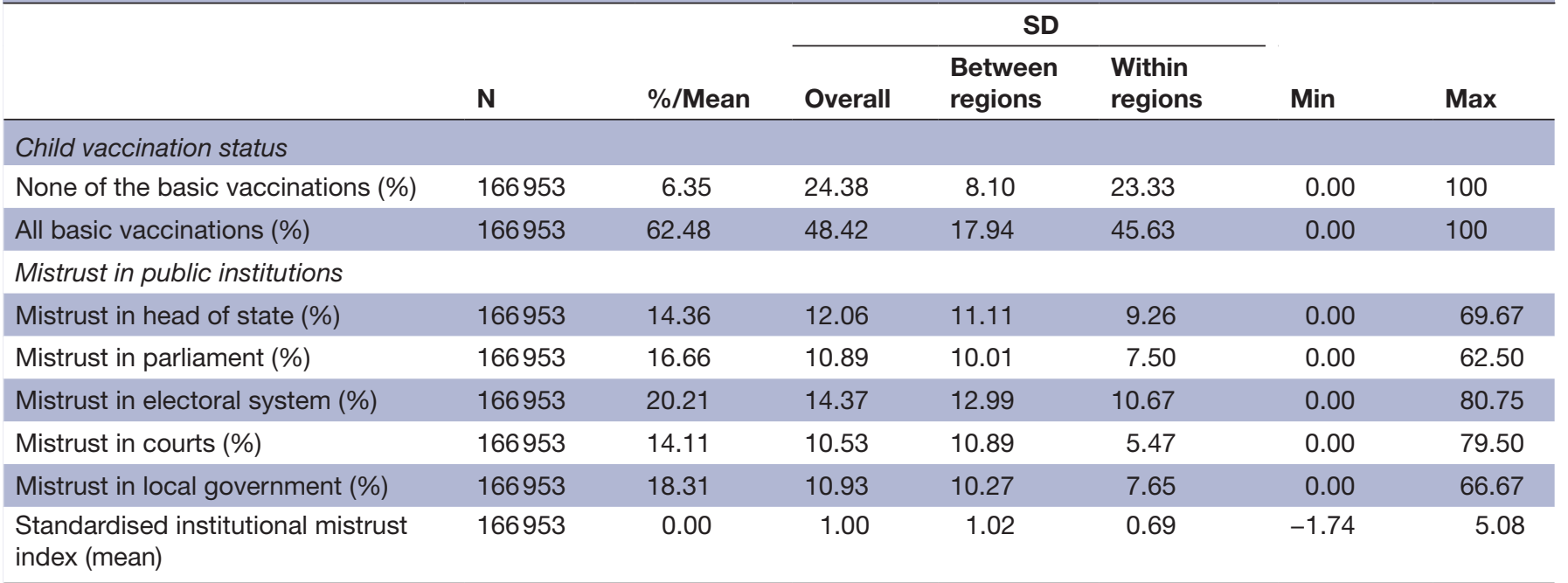

The standardised institutional mistrust index was constructed using a principal component analysis method that combined the level of mistrust in the head of state, parliament, electoral system, courts and local government (see online supplemental appendix 2).

About $6 \%$ of the children aged 12-59 months in our sample had not received any of the eight basic vaccinations while $62 \%$ had received all. Institutional mistrust at the subnational regional level was highest with respect to the electoral system (with $20 \%$ of the population indicating having 'no trust at all'), followed by mistrust in local government (18\%), parliament (17\%), head of state $(14 \%)$ and courts (14\%). The SD measured from within subnational region variations ranged between 7.5 (mistrust in parliament) and 10.7 (electoral system) percentage points indicating considerable temporal variation in mistrust levels within the same subnational region. Subnational region level child vaccination status and the degree of public mistrust in each of the 22 countries are shown in figure 1, based on the most recent available data for each country. Compared with countries in Eastern Africa, institutional mistrust in public institutions was generally higher among countries in West Africa and Southern Africa. The map further indicates that child vaccination coverage was higher in Eastern Africa than in West and Southern Africa. However, the maps also highlight considerable subnational variations in levels of mistrust and vaccination coverage. Using these data, we found that regional mistrust in public institutions was positively correlated with the percentage of children having received none of the basic vaccinations (correlation coefficient: 0.23 ; $\mathrm{p}<0.001$ ) and negatively correlated with the percentage of children having received all eight basic vaccinations (correlation coefficient: $-0.26 ; \mathrm{p}<0.001$ ) (online supplemental appendix 4).

Our multivariable fixed effects logistic regressions indicated that institutional mistrust was strongly associated with child vaccination status (table 2). A 1 SD increase in the institutional mistrust index was associated with a $10 \%$ increase in the likelihood that the child had not received any of the basic vaccines (95\% CI of ORs: 1.03 to 1.18). Similarly, a 1 SD increase in the institutional mistrust index was associated with a $6 \%$ decrease in the likelihood that the child had received all basic vaccinations (95\% CI: 0.92 to 0.97 ).

Institutional mistrust was negatively associated with the likelihood that a child had received each of the eight individual basic vaccinations (figure 2). The ORs ranged between 0.92 and 0.96 indicating that a $1 \mathrm{SD}$ increase in the institutional mistrust index decreased the likelihood that the child had received a specific vaccine by $4 \%-8 \%$.

Each of the five dimensions of institutional mistrust were individually correlated with child vaccination status (table 3). The negative association with child vaccination status was strongest for mistrust in courts, the parliament and local government. The ORs in table 3 quantify the change in child vaccination status when the mistrust in the share of population in the subnational region changes from $0 \%$ to $100 \%$. Thus, a $10 \%$ point increase in mistrust in parliament, for instance, was associated with an $11 \%$ increase in the likelihood that the child had not received any of the basic vaccines and with a $3.7 \%$ decrease in the likelihood that the child had received all basic vaccinations.

Results from several robustness analyses are provided in the online supplemental appendix. Our regression results were robust to different subsets of controls and fixed effects (online supplemental appendix 5). Controlling for maternal religion had no influence on our main findings (online supplemental appendix 6). In contrast to institutional mistrust, we found that mistrust in relatives and people in general was not significantly related to child vaccination status (online supplemental appendix 7). While the proxy for local mistrust in medicine was strongly and negatively correlated with child vaccination status, adding it as an additional control in our main regression models did not alter the estimated 

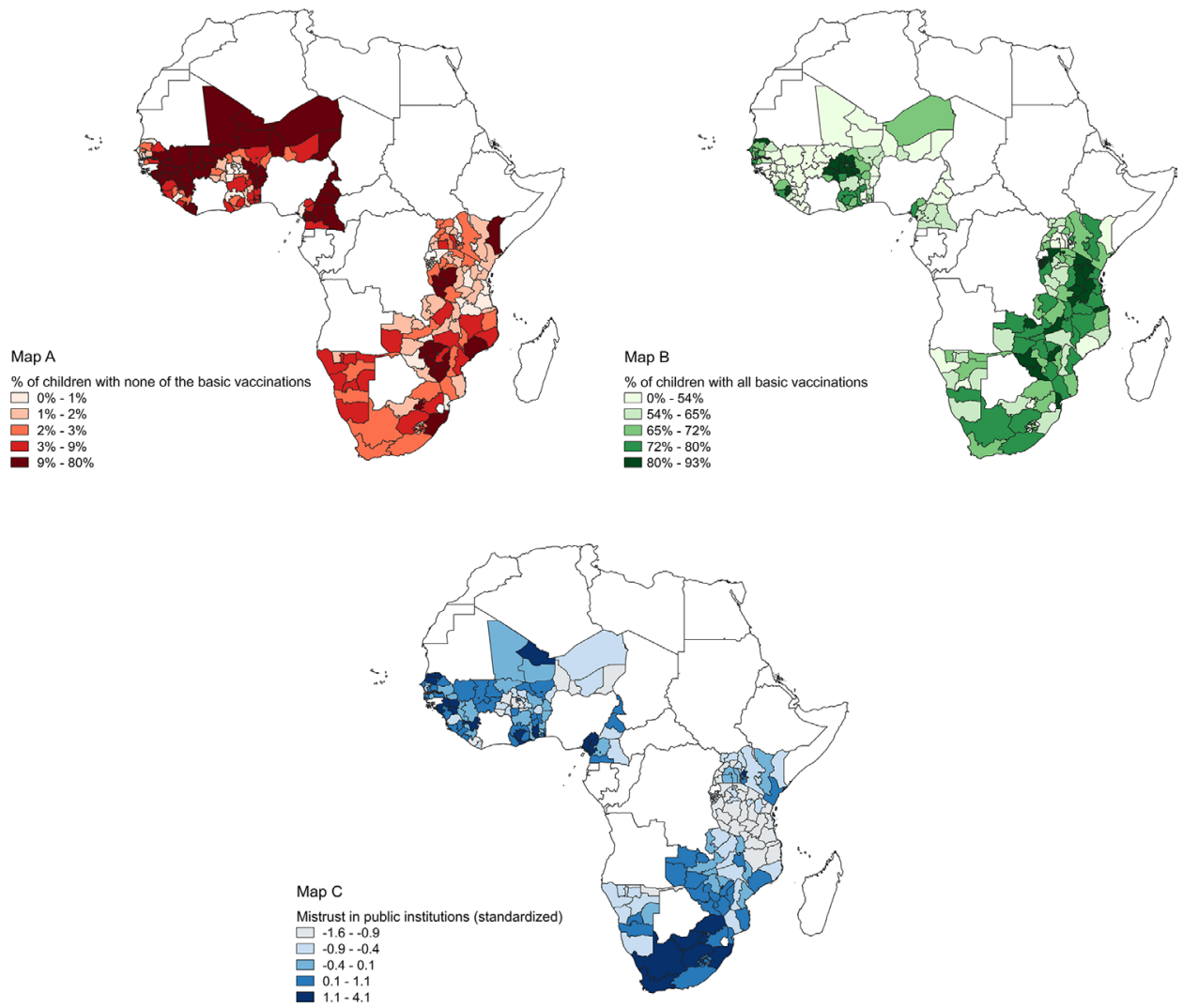

Figure 1 Maps A and B present the child vaccination status from the most recent DHS survey used in this study. Table A.1 in online supplemental appendix 1 provides on overview of the DHS surveys used by country. Categories represent quintiles. Map C presents the degree of mistrust in public institutions from the most recent Afrobarometer survey used in this study. Table A.7 in online supplemental appendix 2 provides an overview of the Afrobarometer surveys used by country. Categories represent quintiles of the mistrust in public institutions index we constructed (online supplemental appendix 2 provides detailed information). Using the data presented in these maps, we found that regional mistrust in public institutions was positively correlated with the percentage of children having received none of the basic vaccinations (correlation coefficient: 0.23 ; $p<$ 0.001 ) and negatively correlated with the percentage of children having received all basic vaccinations (correlation coefficient: $-0.26 ; p<0.001)$.

ORs for mistrust in public institutions (online supplemental appendix 7). Restricting the sample to children whose mother had lived in the same cluster at least since the child's birth resulted in near-identical regression estimates (online supplemental appendix 8). The estimated association between institutional mistrust and child vaccination status remained stable when individual countries and regions (Central, Eastern, Southern and West Africa) were omitted from the sample (online supplemental appendix 9) and when children with missing vaccination information were treated as not having received any vaccine (online supplemental appendix 10). Our

Table 2 Regression results for the association between institutional mistrust and child vaccination status

\begin{tabular}{|c|c|c|c|c|c|c|}
\hline & \multicolumn{3}{|c|}{ None of the basic vaccinations } & \multicolumn{3}{|c|}{ All basic vaccinations } \\
\hline & $\mathbf{N}$ & $\mathrm{OR}^{*}(95 \% \mathrm{Cl})$ & $P$ value & $\mathbf{N}$ & $\mathrm{OR}^{\star}(95 \% \mathrm{Cl})$ & $P$ value \\
\hline $\begin{array}{l}\text { Standardised institutional } \\
\text { mistrust index }\end{array}$ & 162823 & $1.10(1.03$ to 1.18$)$ & 0.003 & 166953 & $0.94(0.92$ to 0.97$)$ & 0.00002 \\
\hline
\end{tabular}

Data are ORs from logistic regression that regressed child vaccination status on a standardised index of public mistrust (continuous measure). Each OR quantifies the associated change in the likelihood that the child had not received any of the basic vaccinations or the likelihood that the child had received all basic vaccinations when public mistrust is increased by 1 SD. Sample restricted to children aged 12-59 months. Subnational regions without variation in the outcome variable were omitted from the sample resulting in a different number of observations $(\mathrm{N})$ across the models.

${ }^{*}$ Adjusted for differences in child's age (binary variables for different age cohorts), sex, birth-order and birth-interval; maternal age and age at which her first child was born; maternal and paternal level of education; household wealth, demographics and location (rural/ urban); time-invariant subnational region characteristics (subnational region fixed effects) and variation across DHS survey years (survey-year fixed effects). We further controlled for access to and utilisation of healthcare services at the household and DHS cluster level.

DHS, Demographic and Health Surveys. 
Odds ratio

Outcome (N)

$(95 \% \mathrm{CI})$

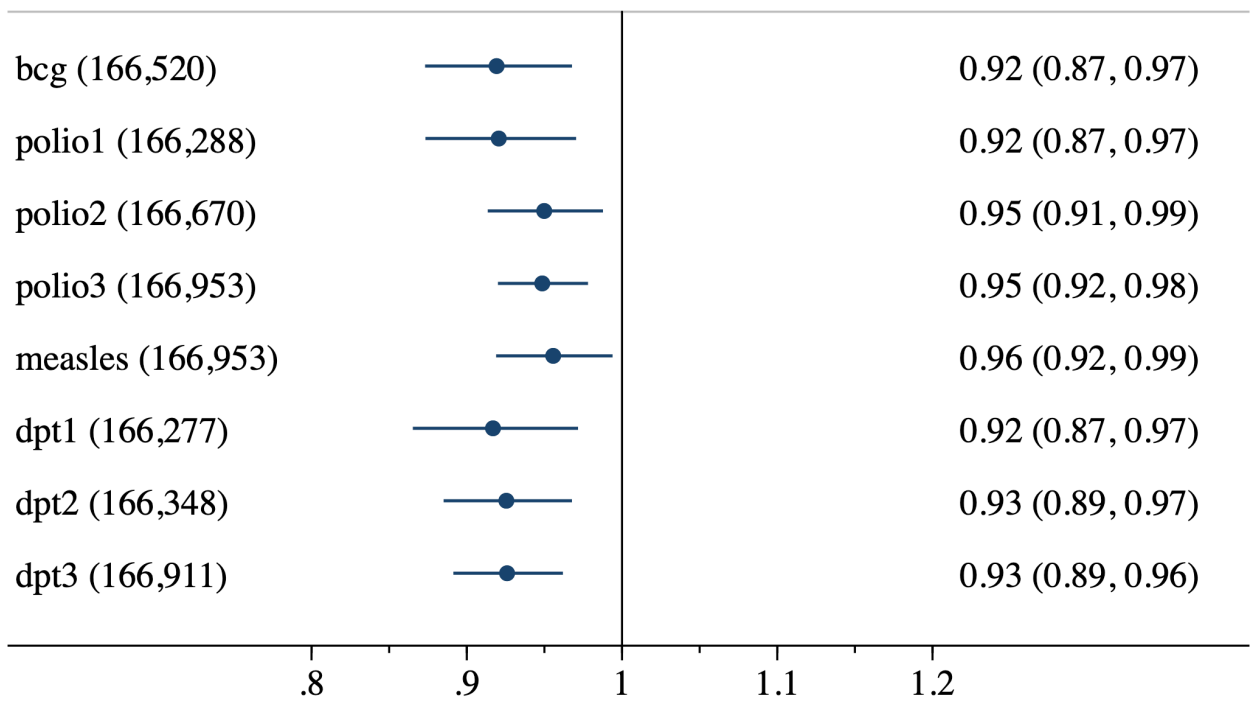

Odds ratio for a one-standard deviation increase in institutional mistrust

Figure 2 Regression results for the association between institutional mistrust and vaccine type. Data are ORs from logistic regressions that regressed child's vaccine status on a standardised index of public mistrust (continuous measure). Each OR quantifies the associated change in the likelihood that the child had received the vaccine when public mistrust is increased by $1 \mathrm{SD}$. dpt $1=$ first dose of diphtheria, pertussis and tetanus vaccine; dpt2=second dose of diphtheria, pertussis and tetanus vaccine; dpt3=third dose of diphtheria, pertussis and tetanus vaccine; measles=one dose of measles-containing vaccine; polio1=first dose of polio vaccine; polio2=second dose of polio vaccine; polio3=third dose of polio vaccine. Sample restricted to children aged 12-59 months. Subnational regions without variation in the outcome variable were omitted from the sample resulting in a different number of observations $(\mathrm{N})$ across the models. *Adjusted for differences in child's age (binary variables for different age cohorts), sex, birth-order and birth-interval; maternal age and age at which her first child was born; maternal and paternal level of education; household wealth, demographics and location (rural/urban); time-invariant subnational region characteristics (subnational region fixed effects) and variation across the Demographic and Health Surveys (DHS) survey years (survey-year fixed effects). We further controlled for access to and utilisation of healthcare services at the household and DHS cluster level.

results were also robust to the use of sampling weights, including those correcting for multicountry survey representativeness (online supplemental appendix 11).
Finally, our findings were robust to aggregating the analysis at the first sub-national administrative level (online supplemental appendix 12).

Table 3 Regression results for the association between different dimensions of institutional mistrust and child vaccination status

\begin{tabular}{|c|c|c|c|c|c|c|}
\hline & \multicolumn{3}{|c|}{ None of the basic vaccinations } & \multicolumn{3}{|c|}{ All basic vaccinations } \\
\hline & $\mathbf{N}$ & $\mathrm{OR}^{\star}(95 \% \mathrm{Cl})$ & $P$ value & $\mathbf{N}$ & $\mathrm{OR}^{\star}(95 \% \mathrm{Cl})$ & $P$ value \\
\hline Mistrust in head of state & 162823 & 1.61 (0.1 to 2.60$)$ & 0.05 & 166953 & $0.77(0.60$ to 0.98$)$ & 0.04 \\
\hline Mistrust in parliament & 162823 & 2.14 (1.15 to 3.97$)$ & 0.02 & 166953 & $0.63(0.50$ to 0.81$)$ & 0.0003 \\
\hline Mistrust in electoral system & 162823 & 1.60 (1.08 to 2.37$)$ & 0.02 & 166953 & $0.80(0.67$ to 0.95$)$ & 0.01 \\
\hline Mistrust in courts & 162823 & 2.65 (1.34 to 5.23$)$ & 0.005 & 166953 & $0.37(0.25$ to 0.55$)$ & 0.000001 \\
\hline Mistrust in local government & 162823 & 2.06 (1.16 to 3.65$)$ & 0.01 & 166953 & 0.66 (0.51 to 0.85$)$ & 0.001 \\
\hline
\end{tabular}

Data are ORs from a logistic regression that regressed child vaccination status on a dimension of public mistrust (continuous measure). The ORs quantify the change in child vaccination status when mistrust in the subnational region changes from $0 \%$ to $100 \%$. Sample restricted to children aged 12-59 months. Subnational regions without variation in the outcome variable were omitted from the sample resulting in a different number of observations $(\mathrm{N})$ across the models.

*Adjusted for differences in child's age (binary variables for different age cohorts), sex, birth-order and birth-interval; maternal age and age at which her first child was born; maternal and paternal level of education; household wealth, demographics and location (rural/urban); time-invariant subnational region characteristics (subnational region fixed effects) and variation across DHS survey years (survey-year fixed effects). We further controlled for access to and utilisation of healthcare services at the household and DHS cluster level.

DHS, Demographic and Health Surveys. 


\section{DISCUSSION}

Vaccine hesitancy is considered as one of the most important threats to global health. ${ }^{42}$ A less explored dimension of vaccine hesitancy relates to a lack of trust in public institutions that oversee vaccination campaigns and healthcare services. To our knowledge, this study is the first to quantify the role of institutional mistrust on child vaccination uptake based on a child-level analysis in a large number of African countries. We found that increases in mistrust in public institutions were strongly and negatively correlated with child vaccination uptake, even after controlling for differences in a number of child, caregiver, household and community characteristics, including access to and utilisation of healthcare services.

These findings resonate with various qualitative reports highlighting the role of institutional trust in vaccination hesitancy across Africa. ${ }^{28-32}$ They are also in line with a quantitative study from the Democratic Republic of Congo during the Ebola disease epidemic that documented associations between low trust in the government and Ebola vaccine acceptance. ${ }^{33}$ We advanced this literature in two ways. First, we considered a large number of countries from Africa- a continent that is lagging behind in terms of child vaccination coverage. ${ }^{4}$ Second, while gathering experimental evidence on this topic is challenging, we moved beyond simple associations by using subnational region and survey year fixed effects models that further controlled for differences in various child, parent, household and community characteristics. We also conducted a battery of sensitivity checks that confirmed our findings. An important limitation relates to the fact that we measure mistrust at the subnational region level, which may mask heterogeneity in trust and vaccination coverage within subnational regions. New survey tools have been developed to assess the nature and scale of vaccination hesitancy, ${ }^{43} 44$ but so far the application to African countries has been restricted to cross-country comparisons. ${ }^{15}$ Incorporating these types of questions directly into the DHS would allow researchers to measure both trust and vaccination coverage at the household level.

Most of the world's unvaccinated or undervaccinated children are located in Africa. ${ }^{4}$ Aggregate numbers on vaccination coverage hide large subnational disparities, ${ }^{6}$ some of which could be due to high degrees of institutional mistrust. Recognising the extent to which a lack of institutional trust jeopardises vaccination campaigns is crucial to improve immunisation rates. An obvious implication of these findings is to strengthen ongoing communication efforts on the benefits of vaccines and to address myths and misunderstandings. However, awareness raising is unlikely to be sufficient unless trust and confidence in those providing the information and delivering vaccination services are increased. ${ }^{45}$ This calls for empowering local authorities with financial and technical capacity and with communication strategies to address mistrust. ${ }^{46}$ For instance, the CORE Group partner project in North East Nigeria has managed to reduce vaccine hesitancy by constructing trusted spaces for community dialogue and engagement in a challenging environment. ${ }^{47}$ Lessons from other successful campaigns highlight the importance of engaging with trusted political or religious leaders. ${ }^{45} 48$ Moreover, a better understanding of the origins of mistrust in relation to vaccine hesitancy is needed to guide specific policies in each country. African economic development has been deeply rooted in the colonial past and in the history of slave trade. ${ }^{49}$ Emerging evidence suggests that even today, these past experiences may explain vaccine hesitancy and ineffectiveness of health interventions in the continent. ${ }^{41}$ Further research is needed to identify the mechanisms through which the determinants of vaccine hesitancy such as the level of education, socioeconomic status, media exposure and social norms interact with public mistrust. ${ }^{15} 5051$ The role of social networks also needs scrutiny, particularly in the context of COVID-19, where restricted mobility and physical isolation have provided online communities a new platform for spreading antivaccination views. ${ }^{52} 53$ Trust-smart policies are needed to protect the 2.6 million under- 5 children estimated to be at risk of dying due to vaccine-preventable diseases by $2030 .^{54}$

\section{Author affiliations}

${ }^{1}$ Institute of Development Policy (IOB), University of Antwerp, Antwerpen, Belgium ${ }^{2}$ Centre for Institutions and Economic Performance (LICOS), University of Leuven, Leuven, Belgium

${ }^{3}$ Research Foundation Flanders (FWO), Brussels, Belgium

${ }^{4}$ Development Strategy and Governance Division, International Food Policy

Research Institute, Addis Ababa, Ethiopia

${ }^{5}$ Economics, Institute of Economic and Social Research (IRES), Louvain Institute of Data Analysis and Modeling in Economics and Statistics (LIDAM), UCLouvain, Louvain-la-Neuve, Belgium

${ }^{6}$ Economics, Lancaster University Management School, Lancaster University, Lancaster, UK

${ }^{7}$ Fonds de la Recherche Scientifique (FNRS), Brussels, Belgium

Twitter Jean-Francois Maystadt @jfmaystadt

Acknowledgements We thank Kaleab Baye and Andrea Guariso for useful comments.

Contributors J-FM conceived the study. All authors contributed to the study design. NS performed data analysis, in consultation with $\mathrm{KH}$ and J-FM. NS, KH and J-FM wrote the manuscript. All authors approved the final version of the manuscript.

Funding This study was funded by Fonds Wetenschappelijk Onderzoek (12W8320N).

Map disclaimer The depiction of boundaries on the map(s) in this article does not imply the expression of any opinion whatsoever on the part of BMJ (or any member of its group) concerning the legal status of any country, territory, jurisdiction or area or of its authorities. The map(s) are provided without any warranty of any kind, either express or implied.

\section{Competing interests None declared.}

Patient consent for publication Not required.

Ethics approval Information on child vaccination status came from the Demographic and Health Surveys (DHS). The protocols and questionnaires of DHS surveys have been reviewed and approved by ICF Institutional review board (IRB) and the IRB of the host countries. Ethical review documentation are available on request at https://dhsprogram.com/What-We-Do/Protecting-the-Privacy-of-DHSSurvey-Respondents.cfm.

Provenance and peer review Not commissioned; externally peer reviewed.

Data availability statement Data may be obtained from a third party and are not publicly available. Data can be freely obtained from the DHS program (https:// 
dhsprogram.com/Data/) and the Afrobarometer (https://www.afrobarometer. org/). Data are protected by copyright. All codes necessary to replicate the results presented in the manuscript will be made available on our personal websites.

Supplemental material This content has been supplied by the author(s). It has not been vetted by BMJ Publishing Group Limited (BMJ) and may not have been peer-reviewed. Any opinions or recommendations discussed are solely those of the author(s) and are not endorsed by BMJ. BMJ disclaims all liability and responsibility arising from any reliance placed on the content. Where the content includes any translated material, BMJ does not warrant the accuracy and reliability of the translations (including but not limited to local regulations, clinical guidelines, terminology, drug names and drug dosages), and is not responsible for any error and/or omissions arising from translation and adaptation or otherwise.

Open access This is an open access article distributed in accordance with the Creative Commons Attribution Non Commercial (CC BY-NC 4.0) license, which permits others to distribute, remix, adapt, build upon this work non-commercially, and license their derivative works on different terms, provided the original work is properly cited, appropriate credit is given, any changes made indicated, and the use is non-commercial. See: http://creativecommons.org/licenses/by-nc/4.0/.

\section{ORCID iDs}

Nik Stoop http://orcid.org/0000-0003-0787-8038

Kalle Hirvonen http://orcid.org/0000-0003-2057-1612

Jean-Francois Maystadt http://orcid.org/0000-0001-7832-1056

\section{REFERENCES}

1 Piot P, Larson HJ, O'Brien KL, et al. Immunization: vital progress, unfinished agenda. Nature 2019;575:119-29.

2 Organization WH. Immunization agenda 2030: a global strategy to leave no one behind. WHO, 2019.

3 WHO. World health statistics 2020: monitoring health for the SDGs, sustainable development goals. Geneva: World Health Organization (WHO), 2020.

4 UNICEF, WHO. Progress and challenges with achieving universal immunization coverage. Geneva: UNICEF and World Health Organization (WHO), 2019.

5 UNICEF. Immunization coverage survey data. In: Immunization data. Geneva: UNICEF, 2020.

6 Utazi CE, Thorley J, Alegana VA, et al. Mapping vaccination coverage to explore the effects of delivery mechanisms and inform vaccination strategies. Nat Commun 2019;10:1633.

7 Restrepo-Méndez MC, Barros AJD, Wong KLM, et al. Inequalities in full immunization coverage: trends in low- and middle-income countries. Bull World Health Organ 2016;94:794-805.

8 Mosser JF, Gagne-Maynard W, Rao PC, et al. Mapping diphtheriapertussis-tetanus vaccine coverage in Africa, 2000-2016: a spatial and temporal modelling study. Lancet 2019;393:1843-55.

9 Wariri O, Edem B, Nkereuwem E, et al. Tracking coverage, dropout and multidimensional equity gaps in immunisation systems in West Africa, 2000-2017. BMJ Glob Health 2019;4:e001713.

10 Bangura JB, Xiao S, Qiu D, et al. Barriers to childhood immunization in sub-Saharan Africa: a systematic review. BMC Public Health 2020;20:1-15.

11 Rainey JJ, Watkins M, Ryman TK, et al. Reasons related to nonvaccination and under-vaccination of children in low and middle income countries: findings from a systematic review of the published literature, 1999-2009. Vaccine 2011;29:8215-21.

12 Cooper S, Betsch C, Sambala EZ, et al. Vaccine hesitancy - a potential threat to the achievements of vaccination programmes in Africa. Hum Vaccin Immunother 2018;14:2355-7.

13 Dubé E, Vivion M, MacDonald NE. Vaccine hesitancy, vaccine refusal and the anti-vaccine movement: influence, impact and implications. Expert Rev Vaccines 2015;14:99-117.

14 Gostin LO, Hodge JG, Bloom BR, et al. The public health crisis of underimmunisation: a global plan of action. Lancet Infect Dis 2020;20:e11-16.

15 de Figueiredo A, Simas C, Karafillakis E, et al. Mapping global trends in vaccine confidence and investigating barriers to vaccine uptake: a large-scale retrospective temporal modelling study. Lancet 2020;396:898-908.

16 MacDonald NE, SAGE Working Group on Vaccine Hesitancy. Vaccine hesitancy: definition, scope and determinants. Vaccine 2015;33:4161-4.

17 Cobos Muñoz D, Monzón Llamas L, Bosch-Capblanch X, Munoz DC, Llamas LM. Exposing concerns about vaccination in low- and middle-income countries: a systematic review. Int J Public Health 2015:60:767-80.

18 Peretti-Watel P, Larson HJ, Ward JK, et al. Vaccine hesitancy: Clarifying a theoretical framework for an ambiguous notion. PLoS Curr 2015;7. doi:10.1371/currents. outbreaks.6844c80ff9f5b273f34c91f71b7fc289. [Epub ahead of print: 25 Feb 2015].

19 Larson HJ, Clarke RM, Jarrett C, et al. Measuring trust in vaccination: a systematic review. Hum Vaccin Immunother 2018:14:1599-609.

20 Yaqub O, Castle-Clarke S, Sevdalis N, et al. Attitudes to vaccination: a critical review. Soc Sci Med 2014;112:1-11.

21 Salmon DA, Moulton LH, Omer SB, et al. Factors associated with refusal of childhood vaccines among parents of schoolaged children: a case-control study. Arch Pediatr Adolesc Med 2005;159:470-6.

22 Ozawa S, Stack ML. Public trust and vaccine acceptance-international perspectives. Hum Vaccin Immunother 2013;9:1774-8.

23 Gefenaite G, Smit M, Nijman HW, et al. Comparatively low attendance during human papillomavirus catch-up vaccination among teenage girls in the Netherlands: insights from a behavioral survey among parents. BMC Public Health 2012;12:1-7.

24 Bults M, Beaujean DJMA, Richardus JH, et al. Pandemic influenza A (H1N1) vaccination in the Netherlands: parental Reasoning underlying child vaccination choices. Vaccine 2011;29:6226-35.

25 Moran MB, Frank LB, Chatterjee JS, et al. Information scanning and vaccine safety concerns among African American, Mexican American, and non-Hispanic white women. Patient Educ Couns 2016;99:147-53.

26 Shui IM, Weintraub ES, Gust DA. Parents concerned about vaccine safety: differences in race/ethnicity and attitudes. Am J Prev Med 2006:31:244-51.

27 Smith LE, Amlôt R, Weinman J, et al. A systematic review of factors affecting vaccine uptake in young children. Vaccine 2017;35:6059-69.

28 Abakar MF, Seli D, Lechthaler F, et al. Vaccine hesitancy among mobile pastoralists in Chad: a qualitative study. Int $J$ Equity Health 2018; $17: 167$

29 UNICEF. A question of trust: reasons for refusals revealed by $D R$ Congo study. New York: UNICEF, 2012.

30 Démolis R, Botão C, Heyerdahl LW, et al. A rapid qualitative assessment of oral cholera vaccine anticipated acceptability in a context of resistance towards cholera intervention in Nampula, Mozambique. Vaccine 2018;36:6497-505.

31 Lasco G, Larson HJ. Medical populism and immunisation programmes: illustrative examples and consequences for public health. Glob Public Health 2020;15:334-44.

32 Peprah D, Palmer JJ, Rubin GJ, et al. Perceptions of oral cholera vaccine and reasons for full, partial and non-acceptance during a humanitarian crisis in South Sudan. Vaccine 2016;34:3823-7.

33 Vinck P, Pham PN, Bindu KK, et al. Institutional trust and misinformation in the response to the 2018-19 Ebola outbreak in North Kivu, DR Congo: a population-based survey. Lancet Infect Dis 2019;19:529-36

34 Afrobarometer. Afrobarometer data, Benin, Burkina Faso, Burundi, Cameroon, Ghana, guinea, Kenya, Lesotho, Liberia, Malawi, Mali, Mozambique, Namibia, niger, Senegal, Sierra Leone, South Africa, Tanzania, Togo, Uganda, Zambia, Zimbabwe, rounds 1-7, 2020. Available: http://www.afrobarometer.org

35 ICF. Demographic and health surveys (various). Rockville, MD: ICF: 2004-20.

36 WHO. Harmonizing vaccination coverage measures in household surveys: a primer. Geneva: World Health Organization, 2019.

37 Cooper MW, Brown ME, Hochrainer-Stigler S, et al. Mapping the effects of drought on child stunting. Proc Natl Acad Sci U S A 2019;116:17219-24.

38 Younsi M, Chakroun M. Does social capital determine health? empirical evidence from Mena countries. Soc Sci J 2016;53:371-9.

39 Wagner Z, Heft-Neal S, Bhutta ZA, et al. Armed conflict and child mortality in Africa: a geospatial analysis. Lancet 2018;392:857-65.

40 Gardiner JC, Luo Z, Roman LA. Fixed effects, random effects and Gee: what are the differences? Stat Med 2009;28:221-39.

41 Lowes SR, Montero E. The legacy of colonial medicine in central Africa. Amer Econ Rev. In Press 2021.

42 WHO. Ten threats to global health in 2019. Available: https://www. who.int/news-room/feature-stories/ten-threats-to-global-health-in2019 [Accessed 20 Jun 2020].

43 Opel DJ, Mangione-Smith R, Taylor JA, et al. Development of a survey to identify vaccine-hesitant parents: the parent attitudes about childhood vaccines survey. Hum Vaccin 2011;7:419-25. 
44 Larson HJ, Jarrett C, Schulz WS, et al. Measuring vaccine hesitancy: the development of a survey tool. Vaccine 2015;33:4165-75.

45 Larson HJ. Vaccine trust and the limits of information. Science 2016;353:1207-8.

46 Oku A, Oyo-Ita A, Glenton C, et al. Perceptions and experiences of childhood vaccination communication strategies among caregivers and health workers in Nigeria: a qualitative study. PLoS One 2017;12:e0186733.

47 Usman S, Bologna L, Stamidis KV. The core group partners project in North East Nigeria: community engagement strategies to combat skepticism and build trust for vaccine acceptance. Am J Trop Med Hyg 2019;101:68-73.

48 Dubé E, Gagnon D, MacDonald NE, et al. Strategies intended to address vaccine hesitancy: review of published reviews. Vaccine 2015;33:4191-203.
49 Nunn N. The historical roots of economic development. Science 2020;367. doi:10.1126/science.aaz9986. [Epub ahead of print: 27 Apr 2020].

50 Dubé E, Laberge C, Guay M, et al. Vaccine hesitancy: an overview. Hum Vaccin Immunother 2013;9:1763-73.

51 Larson HJ, Jarrett C, Eckersberger E, et al. Understanding vaccine hesitancy around vaccines and vaccination from a global perspective: a systematic review of published literature, 2007-2012. Vaccine 2014;32:2150-9.

52 Johnson NF, Velásquez N, Restrepo NJ, et al. The online competition between pro- and anti-vaccination views. Nature 2020;582:230-3.

53 Wilson SL, Wiysonge C. Social media and vaccine hesitancy. BMJ Glob Health 2020;5:e004206.

54 Madhi SA, Rees H. Special focus on challenges and opportunities for the development and use of vaccines in Africa. Hum Vaccin Immunother 2018;14:2335-9. 